\title{
Techniques and Steps of Project Physical Progress Control through the Comparison of Site Images with Prototype Models
}

\author{
(Literature Review) \\ Ali KATEBI \\ Assistant Professor, Kharazmi University, Engineering faculty, Dept. of Civil Engineering, \\ Mofateh Ave, Tehran, Iran.
}

\begin{abstract}
Evaluation of project physical progress through statistical data prepared by project staff and project managers may cause problems in the quality of the obtained results due to human error and approximations. Therefore, making use of image and video items of as-constructed situation of projects, and their comparison with prototype models can yield detailed reports on the status of project progress. Producing site images in the form of as-built data, image analysis through image processing techniques, and comparison with prototype models is a new and rapidly growing method for preparing project progress reports.
\end{abstract}

Keywords: project physical progress, as-constructed visual status, project prototype models, visualization

\section{Introduction}

In construction industry, project progress control is considered as one of the most significant success factors in projects. Currently, identifying potential deviations from efficiency and productivity in the field of construction activities is of great importance to project managers. This has been taken into consideration for achieving effective preventive measures for avoiding, or at least minimizing, these deviations.

To monitor project progress, project control processing performed by the written data and information prepared by project members are compared with the prototype data that are usually defined as an approximation of project. It is evident that both the prototype and as-constructed data are approximate and can be misleading due to various reasons such as not being based on detailed and precise data [1]. In the area of project prototype data preparation, extensive studies have been conducted and significant results have been obtained. Three-dimensional mapping, preparation of building information models (B.I.M.) [2] and development of B.I.M. models to four-dimensional building information models that have linked these models to time dimension, have turned into reliable sources of information to be used as prototype data for designed projects.

On the other hand, numerous studies have been conducted and numerous articles have been presented on how to obtain as-constructed data of projects. Visual techniques, processing 
and comparison with prototype data makes provision of information on project progress a considerably easy and reliable process.

Based on previously conducted studies, the present paper investigates the methods of obtaining manufactory images, their analysis, and finally their comparison with project prototype models for producing reports on project physical progress.

\section{Extraction of three-dimensional elements from images and their comparison with project prototype model}

In this method, images of the intended sights are obtained at first. After extraction, processing, and justification of the intended elements, they are retrieved in three-dimensional mode, and then are compared with the corresponding members in the prototype model.

\subsection{Obtaining site data}

Obtaining images from the construction site is an important preliminary stage in project progress control. Depending on the quality of image data obtained from construction site, image processing yields different progress control results; therefore, effective criteria for installation of cameras and image data management for successful photo processing are among determining success factors in construction industry. First, cameras should be positioned so appropriately that the overlap of construction site views is minimized. If cameras are steady and placed in a proper height, then the chances for collecting consistent and appropriate photos increase. Secondly, the cameras must be programmable and have motion, rotation and zooming capabilities. These motion functions that are coordinated by a scheduling feature allow construction engineers to look freely and record specific activities in a customized way. Thirdly, cameras must be efficient in information transfer. Wireless or wired local area networks with internet connection can be beneficial for transferring data to the main server[3]. 


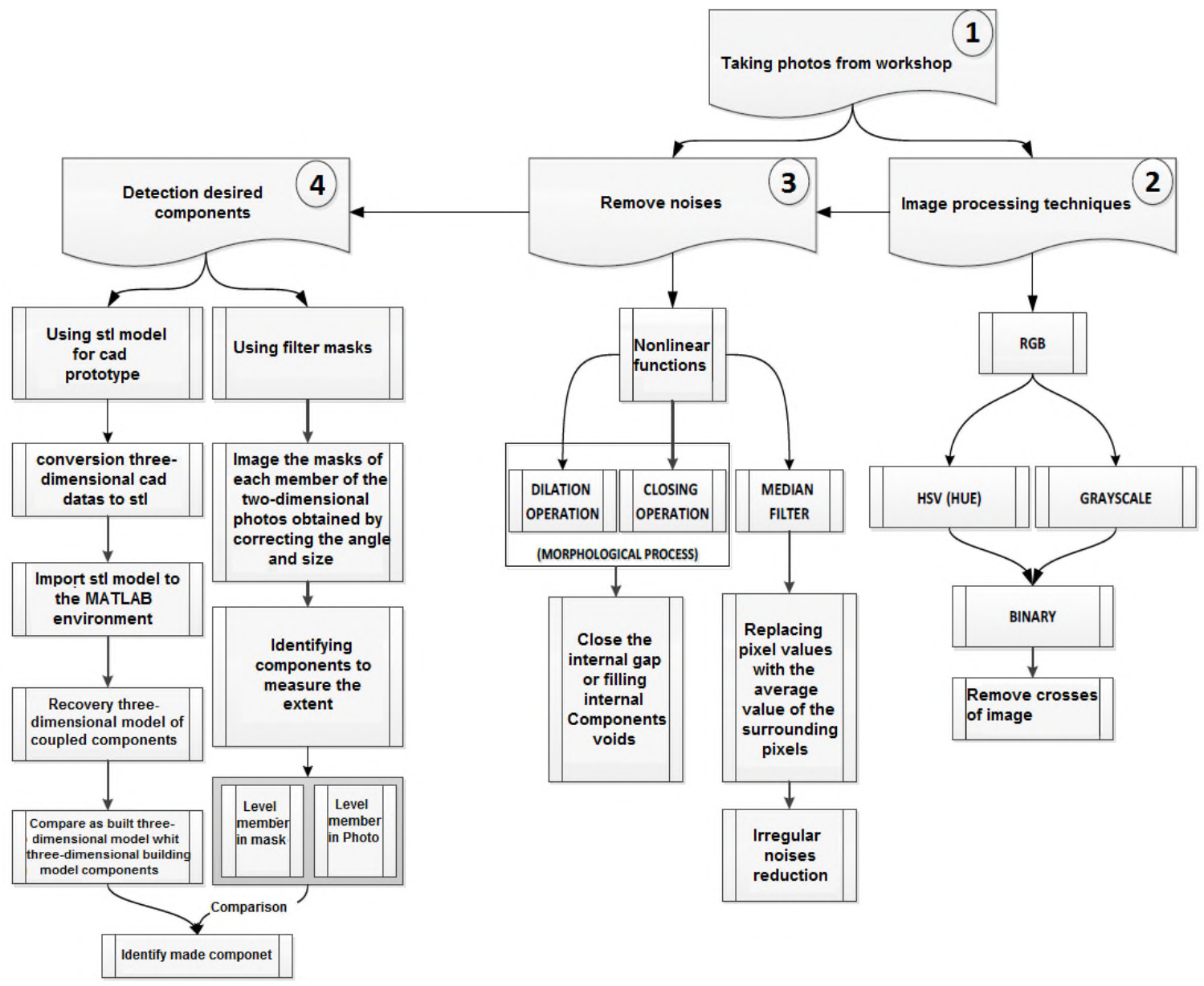

Fig 1: Extraction steps of interested elements of photos and diagnosis element made by comparing the basic model

\subsection{Justification of photos taken from different locations}

For justification of photos, a concept has been presented that can be appropriate for construction site situation with photos of cross-sectional and non-customized coverage. This concept (shown in Fig. 2) combines the structure from motion technique (S.F.M.) [4, 5] through viewing control points in (C.S.R.S.). Mutual registration is resolved by these control points as the construction model should also be available in CSRS. It is worth to note that the construction site has been obtained in several stages and cannot be understood by coverage photos from any position. A barrier may have been created by a construction equipment, or there might be areas that are not available due to the presence of huge items (such as a trailer) or for similar reasons. For high positions, such as a crane or a nearby building, it is likely that a group of photos will not be of proper coverage with one another, or at least have no relationship or similarity for automatic detection. It should also be considered that a construction site is a dynamic environment; that is, the recording condition may change when taking photos or at least between the two stages of 
photography. In addition, it should be considered that for each time interval, new photos must be in the same and fixed coordinate system [6].

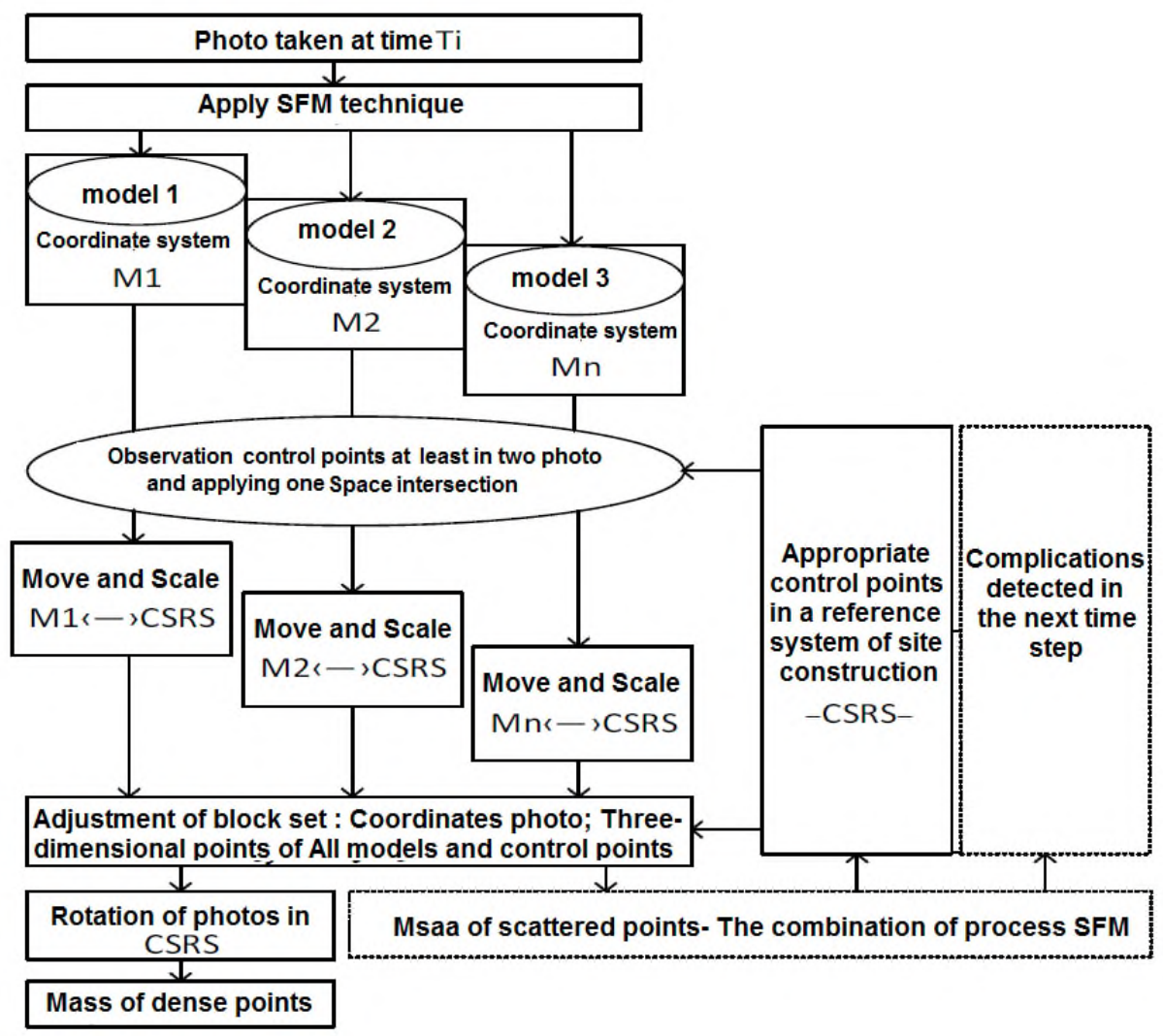

Fig. 2: The method of Justifying Pictures [7]

Based on the requirements, the method shown in Fig. 2 is recommended [7]. Through the SFM process, the proper justification for photos is determined. At least two photos should be selected for the observed control points for each model for absolute calculation with a spatial cut. A bundle block adjustment is formed by combining all models at the final stage. To ensure justification for all photos, control points must be selected well at the start of the construction phase [7].

\subsection{Image Modification}

Image modification includes the removal of unwanted obstacles, noise elimination and filling item holes to improve the resulting images; this prepares the image for comparison with the model. 


\subsubsection{Elimination of unwanted obstacles in photos}

To ensure the successful detection of an item in a construction site, unwanted items present in the image, such as cranes or other construction equipment, must be removed from the images. The colour space of taken photos is the RGB colour space. It is proved that HSV colour space succeeds in equipment detection in construction sites. Hue is less sensitive than RGB to the changing light conditions; this enables Hue to distinguish between different colours. Therefore, Hue is used for generalization of the components that need to be removed. In an HSV image, components with different colours tend to separate from the others as Hue displays the dominant wave length of the colour [8]. As shown in Fig. 3 of a Hue image, the crane has been separated from the rest parts of the image; thus, a simple thresholding process converts the image into a binary image that discards anything other than the crane. In addition, the original RGB image is converted into a grayscale binary image and, as shown in Fig. 3, a thresholding process removes the unwanted equipment from the image, and thus a binary image without the crane is produced. Also, when RGB colour space is transferred to Hue space, a separation algorithm is used to separate the background from the components. To this end, colour threshold is used for separation which is a promising method. Colour threshold, which is based on dividing an image into areas, can extract combinations from a component quickly and easily, if their inherent colours are distinct enough from the colour of the surrounding environment. A large variety of structure components (such as metal, concrete and bricks) that are tracked for progress check of construction projects are independent and easily detectable by their colour [9].

When using colour threshold, determining the optimal threshold values is of prime importance for minimizing separation errors. Using small thresholds increases the possibility of the pixels being actually part of the component in question. However, this can lead to the rejection of some real pixels which show parts of the intended component. Enlarging the threshold results in the increased number of pixels representative of the intended item, but also in increased possibility of pixels not being related to the target item [9]. 
Bulletin de la Société Royale des Sciences de Liège, Vol. 86, 2016, p. 7 - 20

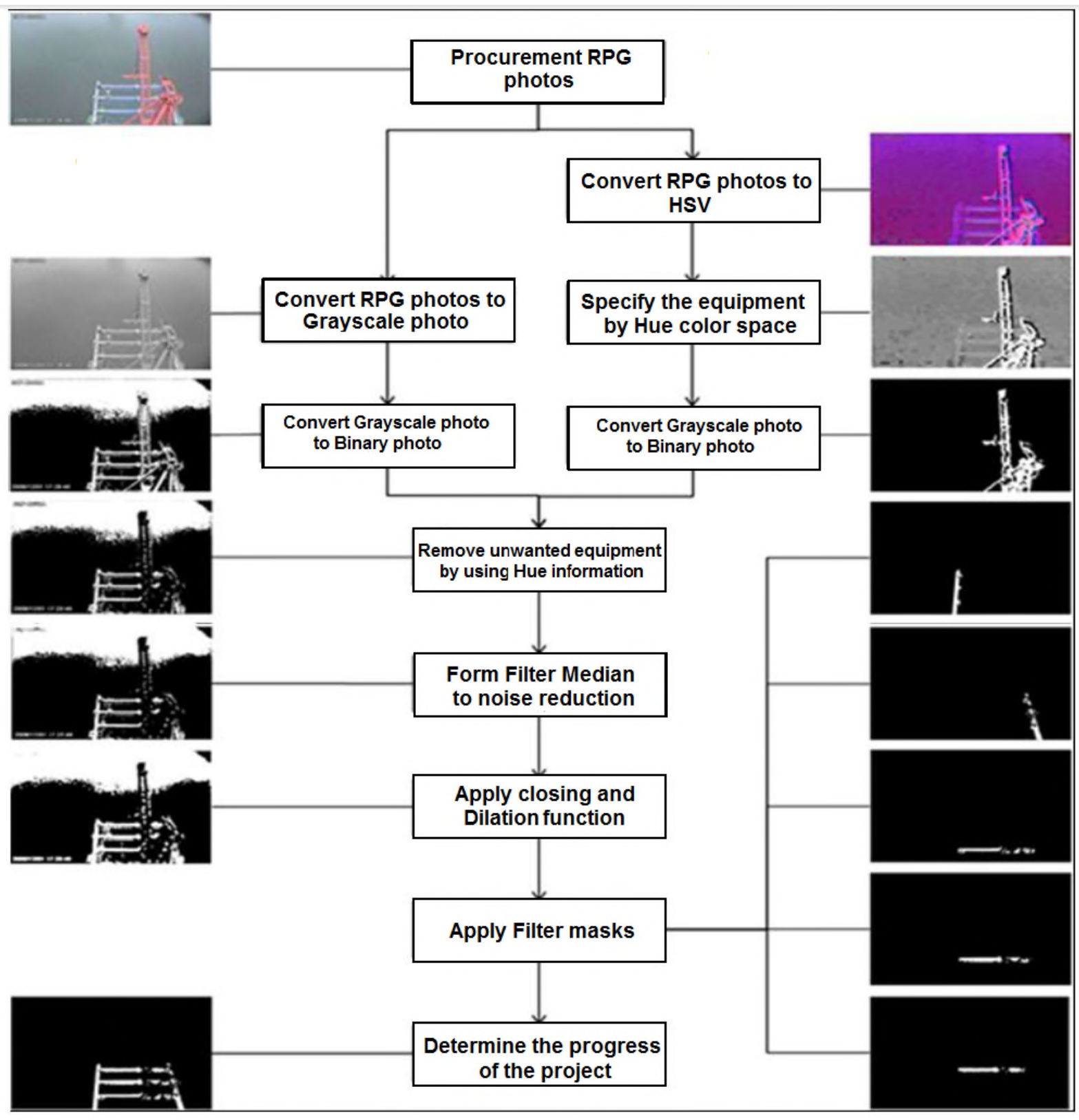

Fig 3: Image analysis to control the progress [8] 


\subsubsection{Removing Noises and Filling the members holes}

Dilation and Closing operators as well as non-linear functions, as a Median Filter [10] are applied for these purposes. Median Filter moves pixel values with average value of environment pixels and is effective in noise reduction. Closing and Dilation operators are forms of morphological operator processing. The former is used for closing the internal gap or filling holes in items, and the latter is used for thickening them. Fig. 3 shows the binary image of a structure after nonlinear functions have been applied [3].

\subsection{Comparison of prototype model with an as-built situation}

\subsubsection{Use method of STL[11] templates for basic models and MATLAB software}

Fig. 4 presents an as-constructed view of a steel structure. Fig. 5(a) shows a complete three-dimensional Cad model of this steel structure. The three-dimensional Cad model has been converted to STL format, and all its parts have been imported to Matlab and have been coupled with extracted three-dimensional data shown in Fig. 5(b). For each component, coupling is performed by checking for the presence of any point mass with a three-dimensional range that includes a depth of $2 \mathrm{~mm}$ from the surface of the components [12].

In case the number of three-dimensional data points within a given component range from the three-dimensional Cad model is above the pre-determined threshold, that component is considered as a constructed component, and is thus retrieved. A component can be considered as constructed, even if only a small number of three-dimensional points are within the shown range. In determining the threshold size, the possibility whether a component is covered by the sensor or quantitative data are proportionately acquired should be taken into consideration.

About the component shown in Fig. 5(c), the number of three-dimensional data points within component range is much higher than the pre-determined threshold; it is thus considered as a constructed component. Fig. 5(d) shows the final three-dimensional as-constructed model [12].

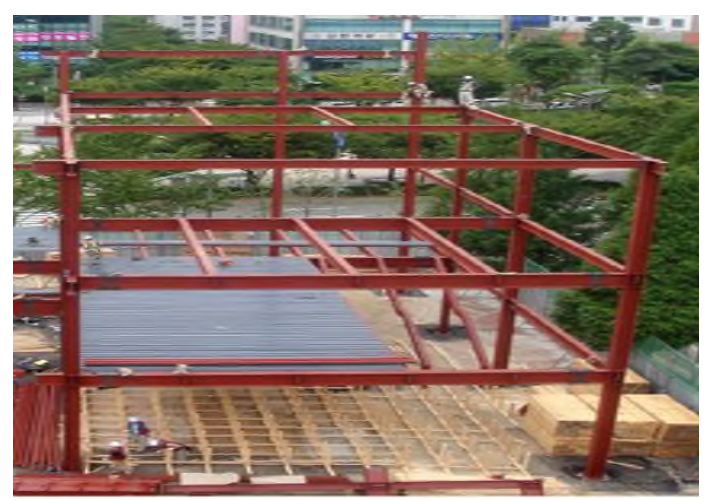

Fig 4: A view of the construction of an under-construction steel structure 
(a)

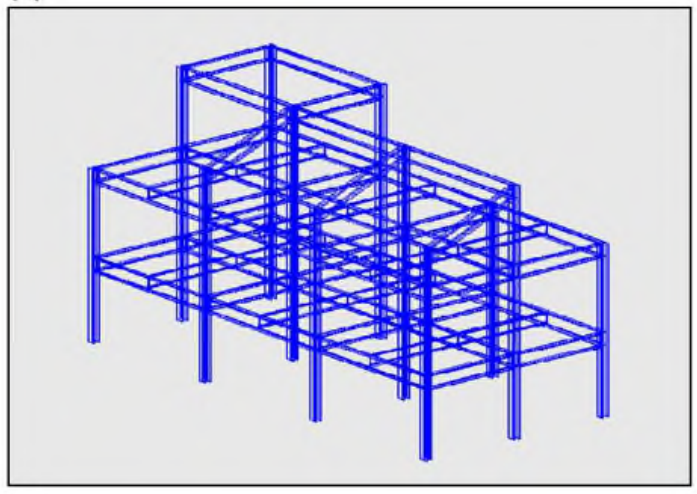

(c)

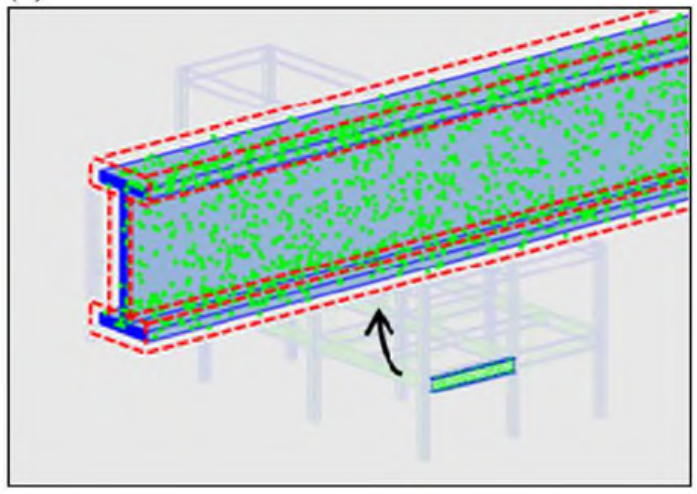

(b)

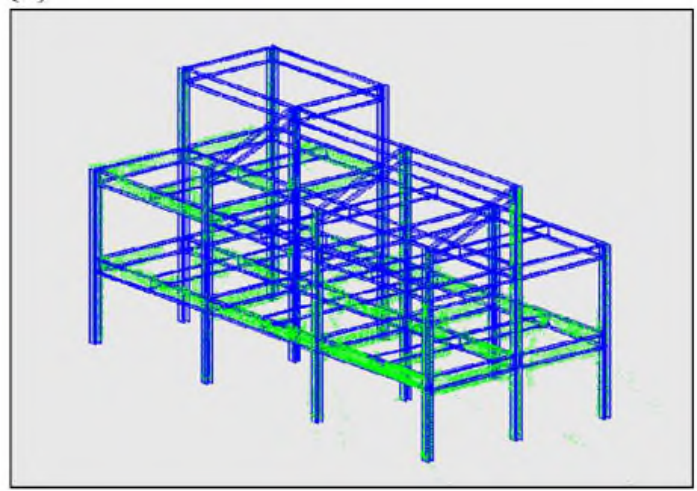

(d)

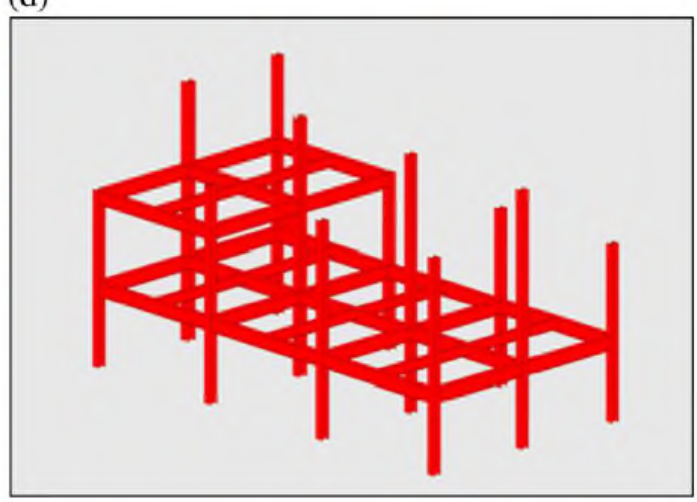

Fig 5: (a) three-dimensional Cad model, (b) coupling a three-dimensional Cad model with threedimensional data like construction, (c) coupling components of three-dimensional model with extracted three-dimensional data, (d) the final three-dimensional Cad model of construction [12].

\subsubsection{Application Method of Filter masks}

In this process, the filter masks of photos based on three-dimensional $\mathrm{Cad}$ is used as reference information to show which part of the image needs further research. Photographic filter masks of a perspective is an image of communication of three-dimensional Cad model. When a three-dimensional model is imaged on the two-dimensional image by correcting the angle and zooming the image, the mask with its configuration is already properly has determined the location of intended member in the image space. In other words, if the mask is placed on the zero and one picture, all unrelated members could be simply filtered. The concept and method of use of masks has already been research and developed [13]. The masks are compared one by one with filtering based photos prepared by HSV, a Median Filter and morphological operators to determine whether or not each member has been made. Comparison is by focusing on a certain range of a component to measure the progress based on level. A simple decision rule like "If member image's surface is more than half the surface of the mask, the member has been installed" can be used. 


\section{Progress assessment using model and three-dimensional image overlay}

In this method, after justification of images and creation of a three-dimensional image model, the prototype model is added as an augmented reality to the image model of the construction site, and then the comparison is made.

\subsection{Calibration of Cameras}

The first task in producing the model and as-constructed image overlay is relating the threedimensional virtual model to two-dimensional images; that is, each point in the threedimensional model such as $\mathrm{p}(\mathrm{x}, \mathrm{y})$ is precisely linked to the image coordinates $\mathrm{P}(\mathrm{u}, \mathrm{v})$. When using periodic images, the camera is positioned in a fixed location in the construction site; therefore, if the camera is set up once, the relation between images and virtual model is determined for all subsequent pictures. As cameras are subject to movement and vibration due to gravity or other external forces such as wind, such adjustments should also be studied and applied. Images and the camera coordinate system are set by a series of parameters; for instance, focal length of camera lens, pixel size, the position of the centre position in the image, and rotation from the actual state [9]. To register the camera, these internal and external camera parameters should be determined. Internal parameters relate the camera coordinate system to the intended coordinate system (including the effective focal length, aspect ratio, the coordinates of image centre, and the coefficient of radial distortion); and external parameters relate the camera coordinate system to a fixed camera coordinate system and identify its position and rotation in the space (Rotation matrix and transmission vector). Autodesk Vis. Software can be employed for automation of the above configurations [1]. 


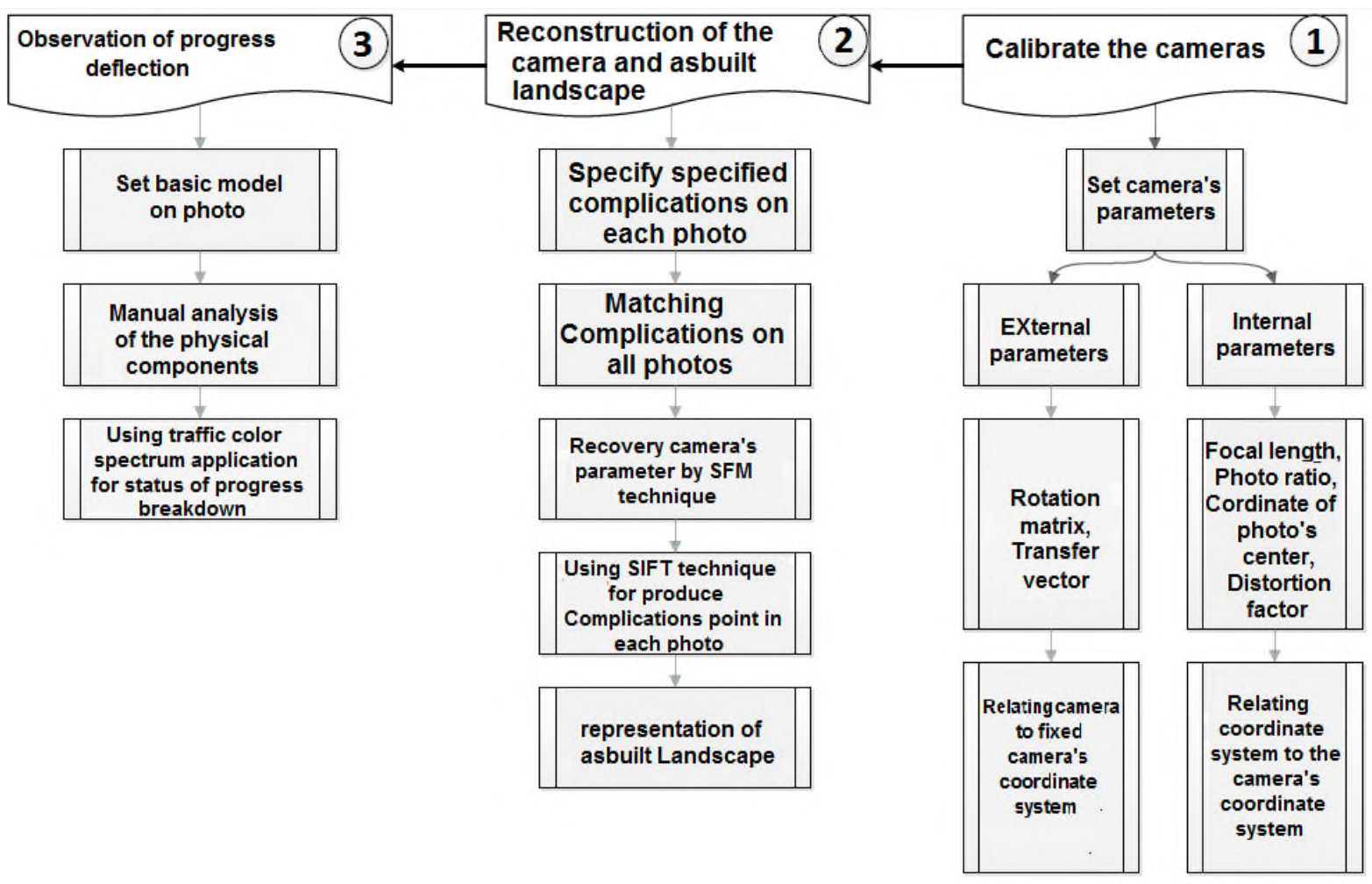

Fig 6: Reconstruction stages of the building and observing distortion by the basic model

\subsection{Reconstruction of camera and as-built view space}

This model requires corrected camera data including external parameters (the relative position and rotation) and internal parameters (focal length and lens distortion). In addition, overlaying the reconstructed image with the designed model needs the absolute position of cameras; therefore, one does not merely rely on camera or equipment such as GPS or wireless detectors to find the location, rotation, and geometry of the image in this system, but some information items are calculated using computer visual techniques on the images.

\subsubsection{Recovery of camera parameters}

First a set of terrains is specified on each image. Then these terrains are coupled on all images and finally SFM method is employed to retrieve the internal and external parameters of the camera. EXIF [14] tags of image files are used to estimate the focal length. However, EXIF tags are not essential when their information is inaccurate. In this system, SFM provides only the relative position of each camera; therefore, another computing system should be used to calculate the transition between the prototype and as-constructed models [1]. A set of three dimensional points of construction condition are connected to the original three-dimensional model so that the sum of least squares errors is minimized. A set of three-dimensional points of the as-constructed 
situation is connected to those in the prototype model so as to minimize the sum of least square errors.

\subsubsection{Finding and coupling the key points}

The first step in reconstruction of as-constructed view is finding the terrain points of each image that can be used to restore the view structure. This can be accomplished by (SIFT) [15, 16] point detection technique which has been examined by researchers in other studies [17] [18] [19] [20]. A $500 \times 500$ pixels thumbnail produces about 1500 to 2000 SIFT combinations.

\subsubsection{Representation of as-built view}

After the as-built view is reconstructed and overlaid on the three-dimensional prototype model, the obtained view should be used for mutual scanning. The following data structure is used to display the as-constructed view:

- A set of key points where each key point includes a three-dimensional position and colour of its own as well as the average of all images that share the same point is obtained;

- A series of cameras when the internal and external parameters are known;

- A mapping between each point and all cameras observing that point;

- A list of camera numbers which have observed that point, the position of that point in local coordinates of the images and SIFT key point index.

When these sets of information are stored, cameras are justified and each camera can display that view from its own viewpoint.

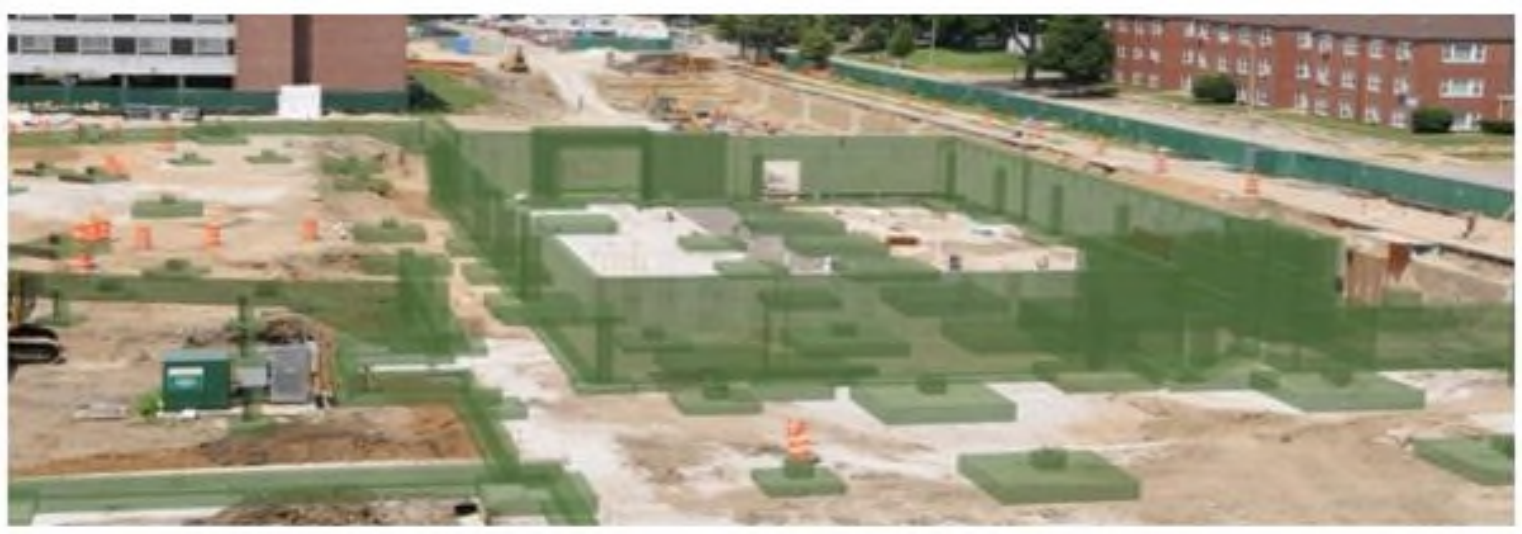

Fig 7: Implémentation of three-dimensional model of the project with one of project photos. As can be seen, some of the foundations and walls have not yet been built [1]. 


\subsection{Observing deviations progress}

When the camera has been set, the prototype model can be overlaid on the image taken by camera. The difference between the prototype model and as-constructed image can be determined easily in this step.

These differences are determined by manual analysis, and the delayed or physically constructed components are identified. Timing deviation of components are formed by the management group, based on the schedule and acquired value analysis (EVA). These deviations can be separated by colours. One method to view project progress deviations is through traffic colour spectrum applications [21] [22]. As observed in Fig. 6, the colour spectrum on the prototype model is used to convey progress deviations. The displayed image shows the real project progress. Behind-schedule, on-schedule, ahead-of-schedule statuses are distinguished by different colours.

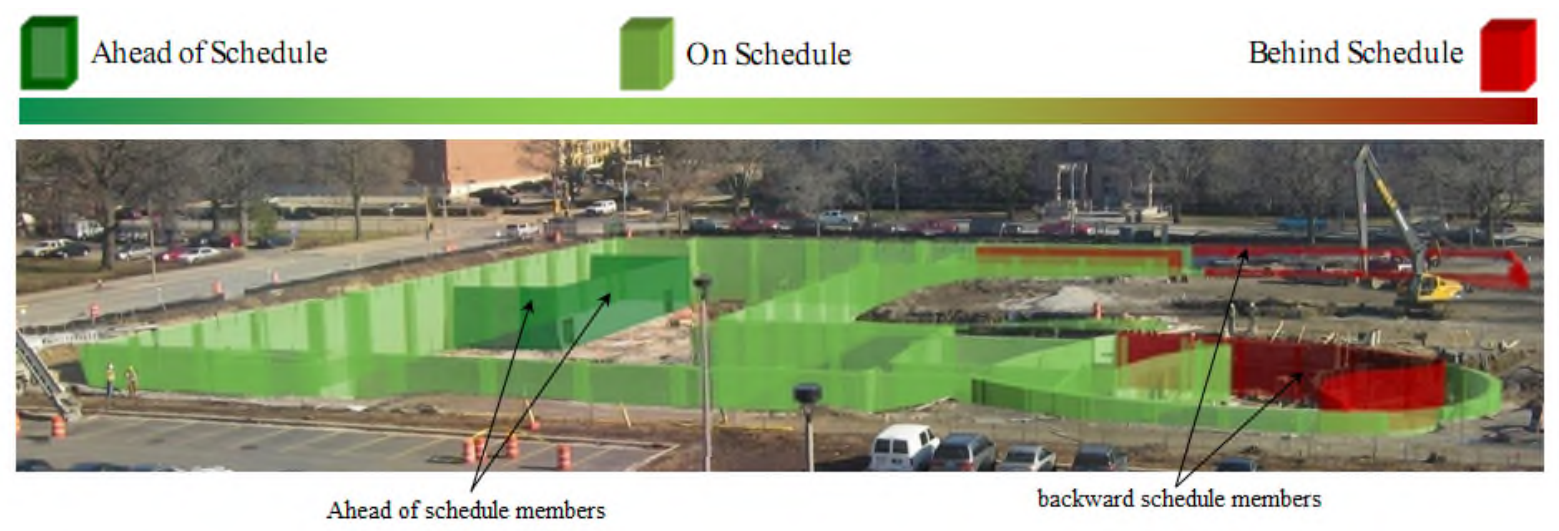

Fig 8: The initial three-dimensional model has taken place on the photo and have been separated by using the traffic color spectrum [23].

\section{Conclusions:}

According to research conducted in the field of building three-dimensional prototype model production and development of these models to four-dimensional models connected to time data, detailed reports can be provided to project managers and operators about project physical progress by providing construction site images and modifying them using image processing techniques to allow comparison with prototype models. Nevertheless, there are many problems in this regard; for instance, the issues related to the justification of the size of elements in images, justification of angles in photos, and obstacles blocking or interfering with visibility, and most importantly the vision field of cameras that links the full coverage of the project to the 
set-up of a variety of cameras, both fixed and mobile. Also, the nature of construction projects is such that the visibility scope of cameras becomes limited as different components and coatings such as walls and ceilings are built. This has discouraged the use and application of images for recording and controlling project progress as a comprehensive way. Also, due to direct relationship between this technique and image processing sciences, the cooperation of project management and construction activists and researchers with image processing researchers can have a beneficial effect on removing the operational barriers of this technique and its application as a comprehensive approach in construction project control.

\section{References:}

1. Savarese, G.-F.F.P.-M.S., A 4-dimensional augmented reality model for automating construction progressing and communication. Journal of Information Technology in Construction, 2009. 14: p. 129-153.

2. Azhar, S., Building Information Modeling (BIM): Trends, Benefits, Risks, and Challenges for the AEC Industry. Leadership and Management in Engineering, 2011. 11(3): p. 241-252.

3. Kim, C., B. Kim, and H. Kim, 4D CAD model updating using image processing-based construction progress monitoring. Automation in Construction, 2013. 35: p. 44-52.

4. Zisserman, R.H.A., Multiple View Geometry in Computer Vision. 2003: Cambridge University Press.

5. wikipedia. Structure from motion. 2016.

6. CENTER, J.R., Best environmental management practice for the building and construction sector. 2012: Institute for Prospective Technological Studies Sustainble Production and Consumption Unit

7. U.Stilla, S.T.A.B.A.B., COMPARISION OF PHOTOGRAMMETRIC POINT CLOUDS WITH BIM BUILDING ELEMENTS FOR CONSTRUCTION PROGRESS MONITORING. The International Archives of the Photogrammetry, Remote Sensing and Spatial Information Sciences, 2014. 3: p. 341-345.

8. Kim, J.Z.H., Using hue, saturation, and value color space for hydraulic excavator. Journal of Computing in Civil Engineering, 2007. 21(4): p. 238-246.

9. Tong, F.F.L., Z.Y.M. Chong, and M.Q.H. Meng. Sensor Fusion and Play Strategy Programming for Micro Soccer Robots. in Robotics and Biomimetics, 2004. ROBIO 2004. IEEE International Conference on. 2004.

10. Huang, T., G. Yang, and G. Tang, A fast two-dimensional median filtering algorithm. IEEE Transactions on Acoustics, Speech, and Signal Processing, 1979. 27(1): p. 13-18.

11. Darvishi. Usable Mime and formats in AutoCAD 2014. 2015.

12. Son, H. and C. Kim, 3D structural component recognition and modeling method using color and $3 D$ data for construction progress monitoring. Automation in Construction, 2010. 19(7): p. 844854.

13. Wu, Y., et al., Object Recognition in Construction-Site Images Using 3D CAD-Based Filtering. Journal of Computing in Civil Engineering, 2010. 24(1): p. 56-64.

14. Manisoft. What's EXIF. 2015.

15. Wikipedia. Scale-invariant feature transform. 
Bulletin de la Société Royale des Sciences de Liège, Vol. 86, 2016, p. 7 - 20

16. Lowe, D.G. Object recognition from local scale-invariant features. in Computer Vision, 1999. The Proceedings of the Seventh IEEE International Conference on. 1999.

17. Lowe, D.G., Distinctive Image Features from Scale-Invariant Keypoints. International Journal of Computer Vision, 2004. 60(2): p. 91-110.

18. Mikolajczyk, K., et al., A Comparison of Affine Region Detectors. International Journal of Computer Vision, 2005. 65(1): p. 43-72.

19. Moreels, P. and P. Perona, A Probabilistic Cascade of Detectors for Individual Object Recognition, in Computer Vision - ECCV 2008: 10th European Conference on Computer Vision, Marseille, France, October 12-18, 2008, Proceedings, Part III, D. Forsyth, P. Torr, and A. Zisserman, Editors. 2008, Springer Berlin Heidelberg: Berlin, Heidelberg. p. 426-439.

20. Tuytelaars, T. and K. Mikolajczyk, Local Invariant Feature Detectors: A Survey. Foundations and Trends ${ }^{\circledR}$ in Computer Graphics and Vision, 2008. 3(3): p. 177-280.

21. Fard, M.G. and F. Peña-Mora, Application of Visualization Techniques for Construction Progress Monitoring, in Computing in Civil Engineering (2007). p. 216-223.

22. Fard, M.G., et al., Visual representation of construction progress monitoring metrics on timelapse photographs. SCIENTIFIC COMMITTEE, 2007: p. 1693.

23. Algohary, T. The integration between $4 D$ simulation, Mobile technology and Augmented reality for automating construction progress monitoring and. 2015. 